\title{
CIRCLE ACTIONS AND FUNDAMENTAL GROUPS FOR HOMOLOGY 4-SPHERES
}

BY

\author{
STEVEN PLOTNICK
}

\begin{abstract}
We generalize work of Fintushel and Pao to give a topological classification of smooth circle actions on oriented 4-manifolds $\Sigma$ satisfying $H_{1}(\Sigma)=0$. We then use these ideas to construct infinite families of homology 4-spheres that do not admit effective circle actions, and whose fundamental groups cannot be 3-manifold groups.
\end{abstract}

0. Introduction. This paper studies several questions related to circle actions on homology 4-spheres, and their fundamental groups. The main result is a construction which yields infinite families of homology 4-spheres $\Sigma^{4}$ (more generally, $\Sigma^{4}$ satisfying $H_{1}(\Sigma ; \mathbf{Z})=0$ ) which do not support effective circle actions, and whose fundamental groups cannot be 3-manifold groups. This construction makes it clear that the gap between fundamental groups of homology 3-spheres and 4-spheres is quite large, as one might expect.

The paper is organized as follows. $\$ 1$ quickly reviews Fintushel's classification of $S^{1}$ actions on simply connected 4-manifolds [F1-F3] and Pao's work on the topological classification [P], and gives a simple generalization to the case where $H_{1}(\Sigma ; \mathbf{Z})=0$. We prove

THEOREM 1.2. Assume that $\Sigma^{4}$ is an oriented, smooth 4-manifold with $H_{1}(\Sigma ; Z)=0$, and that $S^{1}$ acts effectively on $\Sigma$. Then $\Sigma$ may be decomposed as a connected sum of copies of $S^{2} \times S^{2}, \pm \mathbf{C} P^{2}$, and a "spun" homology 3-sphere $M^{3}$. Hence, $\pi_{1}\left(\Sigma^{4}\right) \cong$ $\pi_{1}\left(M^{3}\right)$.

$\$ 2$ describes a construction of homology 4-spheres which generalizes the equivariant construction of $\S 1$. If $K$ is a smooth knot in some homology sphere $M^{3}$, we produce $\Sigma^{4}$ with

$$
\pi_{1}\left(\Sigma^{4}\right) \cong\left\langle\pi_{1}\left(M^{3}-K \times D^{2}\right) \mid 1=m l^{b}=\left[l^{\alpha}, x\right], \forall x\right\rangle,
$$

where $m$ and $l$ are meridian and preferred longitude, $b$ and $\alpha$ are arbitrary integers. $\S 3$ analyzes the case of torus knots in $S^{3}$ using the theory of Seifert manifolds, and we use these results in $\$ 4$ to prove

Received by the editors October 8, 1980 and, in revised form, March 26, 1981

1980 Mathematics Subject Classification. Primary 57S15, 57M99. 
TheOREM 4.1. Let $M^{3}$ be any homology 3-sphere with $\pi_{1} \neq 1$. Pick an $(r, s)$ torus $k n o t$, pick $b$ and $\alpha$ so that $\operatorname{gcd}(\mid$ brs $-1 \mid, \alpha)=\delta \neq 1$, and let $y \delta=|\alpha|$. If $\{r, s, \delta\}=$ $\{2,3,5\}$, assume $y$ is odd. Then the plumbing construction (of \$2) will produce a homology 4-sphere $\Sigma$ with $\pi_{1}(\Sigma) \cong\left\langle\pi_{1} \Sigma(r, s, \delta) * \pi_{1} M\right| i^{y}$ central $\rangle$. $\Sigma$ does not admit an $S^{1}$ action, and $\pi_{1} \Sigma$ is not a 3-manifold group.

This theorem demonstrates a large gap between the groups which arise as fundamental groups of homology spheres in dimensions 3 and 4. Kervaire has shown that the groups which arise as fundamental groups of homology $n$-spheres, $n \geqslant 5$, do not depend on $n$, and are exactly those finitely presented groups $G$ satisfying $H_{1}(G)=H_{2}(G)=0[\mathbf{K}]$. Whether there is a gap between dimensions 4 and 5 seems to be a difficult problem.

This paper is part of the author's doctoral dissertation, written at the University of Michigan under the direction of Professor Frank Raymond. I would like to express my warmest appreciation to Professor Raymond for his guidance and encouragement over the years. I would also like to thank Walter Neumann for many helpful and interesting conversations.

1. Circle actions on 4-manifolds satisfying $H_{1}(\Sigma ; \mathbf{Z})=0$. We first summarize some results in [F1]-[F3] and [P]. Assume that $S^{1}$ acts smoothly and effectively on $\Sigma^{4}$, a homotopy 4-sphere. For a set $X$ in $\Sigma$, let $X^{*}$ denote its image in the orbit space. We have the following information:

(i) The fixed set $F$ is either $S^{2}$ or $S^{0}$ (Smith theory). In the first case, $\Sigma^{*}$ is a homotopy 3-cell with boundary $F^{*}$, and there are no exceptional orbits. In the second case, $\Sigma^{*}$ is a homotopy 3 -sphere.

(ii) Let $E$ denote the exceptional orbit set; i.e., those orbits with nontrivial finite cyclic isotropy subgroup. There are at most two exceptional orbit types. If there is one, then $E^{*} \cup F^{*}$ is a closed arc, and $F^{*}$ is the set of endpoints. If there are two, $E^{*} \cup F^{*}$ is a simple closed curve separated by $F^{*}$ into two open arcs, on each of which the orbit type is constant. Furthermore, if $\mathbf{Z}_{k}$ and $\mathbf{Z}_{p}$ represent the stabilizers of the two exceptional orbit types, $k$ and $p$ are relatively prime.

Thus, given an $S^{1}$ action on $\Sigma^{4}$, we associate to it the orbit data as follows:

(i) $\left\{\Sigma^{*}\right\}$ if there are no exceptional orbits,

(ii) $\left\{\Sigma^{*}, k\right\}$ if there is one orbit of type $\mathbf{Z}_{k}$, or

(iii) $\left\{\left(\Sigma^{*}, E^{*} \cup F^{*}\right), k, p\right\}$ if there are two exceptional orbits of types $\mathbf{Z}_{k}, \mathbf{Z}_{p}$. Fintushel proved [F1, Theorem 1.5] that $S^{1}$ actions on $\Sigma^{4}$ are determined up to weak equivalence by the orbit data.

We shall mostly be interested in the case where there are two exceptional orbit types. In this case, we may construct the action as follows: Let $P^{4}$ be the manifold obtained by plumbing together two copies of $S^{2} \times D^{2}$ at two points. Then $\partial P=T^{3}$ $=S^{1} \times S^{1} \times S^{1}$. Let $S^{1}$ act on one copy of $S^{2} \times D^{2}$ by rotating $S^{2}$ about itself $k$ times, and rotating $D^{2}$ about itself $p$ times. If we reverse the roles of $k$ and $p$ for the other copy of $S^{2} \times D^{2}$, we get an $S^{1}$ action on $P$. The two points where we plumbed are fixed points of the action, and the $S^{2}$ cores of the plumbing represent exceptional orbits of type $\mathbf{Z}_{k}$ and $\mathbf{Z}_{p}$. The quotient space of this action is a solid torus $S^{1} \times D^{2}$, with its core circle representing $E^{*} \cup F^{*}$. 
Now take $\Sigma^{*}$, a homotopy 3-sphere, and remove an open tubular neighborhood of a knot $K$. On $\left(\Sigma^{*}-\left(K \times D^{2}\right)\right) \times S^{1}$, let $S^{1}$ act by translation in the second factor. Now glue $P$ to $\left(\Sigma^{*}-\left(K \times D^{2}\right)\right) \times S^{1}$ by an equivariant diffeomorphism of the boundary $T^{3}$. The result is an $S^{1}$ action on $\Sigma$ with orbit data as described above in (iii).

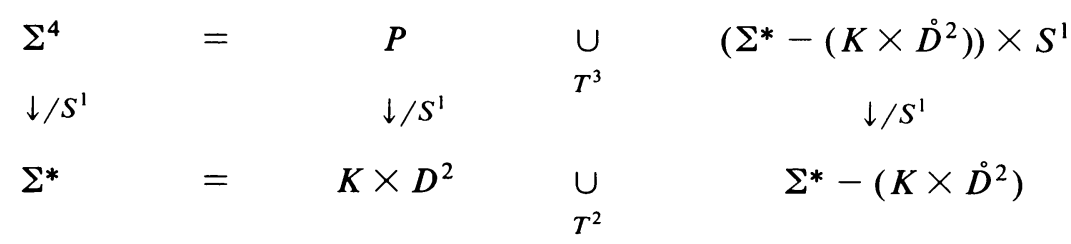

In [P] Pao shows that if $\Sigma^{*}=S^{3}$, then $\Sigma=S^{4}$. In general, $\Sigma^{4}$ is one of $t w o$ possible manifolds obtained by “spinning" $\Sigma^{*}$ (see Definition 1.1).

The situation where $\Sigma^{4}$ is only assumed to be simply connected is somewhat more complicated. The fixed point set will have Euler characteristic $\chi(F)=\chi(\Sigma)=2+$ rank $H_{2}(\Sigma) . \Sigma^{*}$ will be simply connected with $\partial \Sigma^{*}$ a collection of 2 -spheres contained in $F^{*}$, and the closure of $E^{*}$ will be a collection of arcs and knots in $\Sigma^{*}$. The components of $E^{*}$ are open arcs on which orbit types are constant, and the closure of the arcs have endpoints in $F^{*}-\partial \Sigma^{*}$. Again, one constructs plumbing manifolds, using possibly nontrivial $D^{2}$-bundles over $S^{2}$, with $S^{1}$ actions giving tubular neighborhoods of these arcs, knots, boundary components, and isolated fixed points as quotient spaces. Using equivariant connected sums and Pao's result above, Fintushel shows [F3, Theorem 13.2] that, modulo the three-dimensional Poincaré conjecture, $\Sigma^{4}$ must be a connected sum of copies of $S^{4}, \mathbf{C P} P^{2},-\mathbf{C P} P^{2}$, and $S^{2} \times S^{2}$. If we do not assume the Poincaré conjecture, we must worry about "spinning" homotopy 3-spheres, as mentioned above.

Let us now drop the simple connectivity assumption, and assume $H_{1}\left(\Sigma^{4}\right)=0$. Our goal is to show that the same result holds; namely, that $\Sigma^{4}$ is a connected sum of copies of $\pm \mathrm{C} P^{2}$ and $S^{2} \times S^{2}$ with a "spun" homology 3-sphere.

DEFinition 1.1. Let $M^{3}$ be a homology 3-sphere. Pick $* \in M$. Let $\Sigma^{4}$ be one of the two possible manifolds resulting from surgery on $* \times S^{1}$ in $M \times S^{1}$. Then $\Sigma^{4}$ is a homology 4-sphere, and it is called a spun homology sphere.

It is easy to see that both spun homology spheres admit (distinct) circle actions. One might ask whether these two spinnings of $M^{3}$ are distinct manifolds. As far as I know, nothing is known about this except when $M=S^{3}$, in which case $\Sigma^{4}$ is always $S^{4}$. Notice that $\pi_{1}\left(\Sigma^{4}\right) \cong \pi_{1}\left(M^{3}\right)$ in both cases.

THEOREM 1.2. Assume that $\Sigma^{4}$ is an oriented, smooth 4-manifold with $H_{1}(\Sigma ; \mathbf{Z})=0$, and that $S^{1}$ acts effectively on $\Sigma$. Then $\Sigma$ may be decomposed as a connected sum of copies of $S^{2} \times S^{2}, \pm \mathbf{C} P^{2}$, and a spun homology 3-sphere $M^{3}$. Hence $\pi_{1}\left(\Sigma^{4}\right) \cong \pi_{1}\left(M^{3}\right)$.

Proof. Since $E^{*} \cup F^{*}-\partial \Sigma^{*}$ has codimension at least two, we can assume that any element of $\pi_{1}\left(\Sigma^{*}\right)$ is represented by a loop $\alpha$ in $\Sigma^{*}-\left(E^{*} \cup F^{*}\right)$. Now $\left(S^{1}, \Sigma-E \cup F\right)$ is a free circle action, so $\Sigma-(E \cup F) \stackrel{\pi}{\rightarrow} \Sigma^{*}-\left(E^{*} \cup F^{*}\right)$ is a principal $S^{1}$-bundle. Using the lifting property of this fibration and the path-connected fiber, we see that $\alpha$ is the image of a loop in $\Sigma-(E \cup F)$. Hence, 
$\pi_{1}(\Sigma) \stackrel{\pi_{*}}{\rightarrow} \pi_{1}\left(\Sigma^{*}\right)$ is onto, and induces a surjection $0=H_{1}(\Sigma) \rightarrow H_{1}\left(\Sigma^{*}\right)$. Thus, $\Sigma^{*}$ is an oriented 3-manifold, with possibly nonempty boundary, satifying $H_{1}\left(\Sigma^{*}\right)=0$. A standard duality argument now shows that $\Sigma^{*}$ is a homology 3 -sphere $M^{3}$ minus some number of open balls. Boundary spheres correspond to fixed points of the action.

We now show that there are no simple closed curves in $E^{*}$. One way to do this is to use Fintushel's presentation for $\pi_{1}(\Sigma)$ and abelianize [F3, Theorem 10.1]. A more direct way to see that there are no circles in $E^{*}$ is the following. Suppose $K$ is a knot in $E^{*}$ with isotropy subgroup $\mathbf{Z}_{n}$. Find a Seifert surface $G$ with $\partial G=K, G \cap F^{*}=\varnothing$ and $\stackrel{\circ}{G} \cap E^{*}=$ points. Let $\tilde{G}$ and $\tilde{K}$ represent their inverse images in $\Sigma$. Then $\tilde{K}=T^{2}$ and $\tilde{G}$ is a 3-chain with $\partial \tilde{G}=n \cdot \tilde{K}$ (look locally about $K$ to see this). Hence, $\tilde{K}$ is $n$-torsion in $H_{2}(\Sigma)$. But $H_{1}(\Sigma)=0$ implies $H_{2}(\Sigma)$ is free abelian, so $[\tilde{K}]=0 \in$ $H_{2}(\Sigma ; \mathbf{Z})$. Now the map $\Sigma^{4}-\tilde{K} \times D^{2} \rightarrow \Sigma^{*}-K \times D^{2}$ induces a surjection $\mathbf{Z} \cong$ $H_{1}\left(\Sigma-\tilde{K} \times D^{2}\right) \rightarrow H_{1}\left(\Sigma^{*}-K \times D^{2}\right) \cong \mathbf{Z}$ as in previous arguments. But these groups are generated by meridians, and a meridian upstairs is mapped to $n$ meridians downstairs, which is a contradiction.

We have shown that the orbit data is identical to that of $S^{1}$ actions on simplyconnected 4-manifolds, except that $\Sigma^{*}$ satisfies $H_{1}\left(\Sigma^{*}\right)=0$ instead of $\pi_{1}\left(\Sigma^{*}\right)=1$. By [F3, Corollary 10.2], we have $\pi_{1}(\Sigma) \cong \pi_{1}\left(\Sigma^{*}\right)$. In fact, $\Sigma$ can be shown to decompose as a connected sum of copies of $\pm \mathbf{C} P^{2}$ and $S^{2} \times S^{2}$ with a spun $M^{3}$. This is proved in [F3, Theorem 13.2] for the simply-connected case. The argument there carries over in a straightforward fashion to this case, so we omit details.

REMARKS. It is easy to deduce, using the above reasoning, that certain manifolds with boundary cannot support $S^{1}$-actions. Suppose $S^{1}$ acts on an oriented 3manifold $N^{3}$ without fixed points so that the quotient is a closed oriented surface $F$ of genus $g>0$. It is well known that this action bounds, and the standard plumbing construction gives a 4-manifold with rank $H_{1}=2 g$ [O]. Suppose $N^{3}=\partial M^{4}$, where rank $H_{1}(M)<g$. Then the action cannot extend over $M^{4}$-if it did, we would have a surjection $H_{1}(M) \rightarrow H_{1}\left(M^{*}\right)$. But rank $H_{1}\left(M^{*}\right) \geqslant g$, since $F \subset \partial M^{*}$ and a subspace of dimension $g$ must survive under the map $H_{1}\left(\partial M^{*} ; Q\right) \stackrel{i_{*}}{\rightarrow} H_{1}\left(M^{*} ; Q\right)$.

2. Constructing some homology 4-spheres. We have seen that an $S^{1}$ action on a homology 4-sphere consists of the following: take a knot $K \subset M^{3}=$ homology 3-sphere and form $\left(M^{3}-K \times D^{2}\right) \times S^{1} \cup_{T^{3}} P$, where $P$ consists of two copies of $S^{2} \times D^{2}$ plumbed at two points. The manifold $P$ has a circle action with the two plumbing points fixed, and the rest of the 2-sphere cores are comprised of exceptional orbits with stabilizers $\mathbf{Z}_{k}$ and $\mathbf{Z}_{p},(k, p)=1$. The $S^{1}$ action on $(M-K \times$ $\left.D^{2}\right) \times S^{1}$ is in the second factor, and we glue by an equivariant diffeomorphism of $T^{3}$.

Let us now determine all glueings of the above two pieces which yield homology 4-spheres. From now on, $M^{3}$ will denote a homology 3 -sphere, $K \subset M$ will be a smoothly embedded knot, and $P$ will be the plumbing manifold described above.

Suppose we form $\Sigma^{4} \equiv\left(M^{3}-K \times D^{2}\right) \times S^{1} \cup_{T^{3}} P$. Since $P$ deformation retracts to two copies of $S^{2}$ which have been identified at two points, $\pi_{1}(P) \cong H_{1}(P)$ $\cong \mathbf{Z}$, generated by, say, $j$. Also, $H_{1}\left(M-K \times \check{D}^{2}\right) \cong \mathbf{Z}$, generated by a meridian $m$. 
We assume that orientations are chosen so that the linking number $l k(m, K)=+1$. Let $h=* \times S^{1}$, where $*$ is a basepoint on the boundary of $M-K \times D^{2}$.

The Mayer-Vietoris sequence for $\Sigma^{4}$ yields

$$
\begin{aligned}
& \begin{array}{ccccc}
H_{1}\left(T^{3}\right) & \rightarrow & H_{1}\left(\left(M-K \times D^{2}\right) \times S^{1}\right) & \oplus & H_{1}(P)
\end{array} \quad \rightarrow \quad H_{1}(\Sigma) \rightarrow 0 \\
& \mathbf{Z} \oplus \mathbf{Z} \oplus \mathbf{Z} \quad \mathbf{Z} \oplus \mathbf{Z} \quad \mathbf{Z} \\
& \langle m\rangle\langle h\rangle \quad\langle j\rangle
\end{aligned}
$$

In order that $H_{1}(\Sigma)=0$, we must be able to pick a basis $\{x, y, z\}$ for $H_{1}\left(T^{3}\right)=$ $H_{1}(\partial P)$ so that, on the homology level, $x \mapsto m, y \mapsto h, z \mapsto j$. Notice that $\pi_{1}(P) \cong$ $H_{1}(P) \cong \mathbf{Z}$ implies that $x$ and $y$ include trivially in $\pi_{1}(P)$. In these homology considerations, the preferred longitude $l$ of the knot is irrelevant. (By a preferred longitude we mean a simple closed curve on $\partial\left(M-K \times \check{D}^{2}\right)$ which has zero linking with $K$. Then $m$ and $l$ are a basis for the homology of $\partial\left(M-K \times D^{2}\right)$.) But it is not irrelevant when we consider fundamental groups. We have the following:

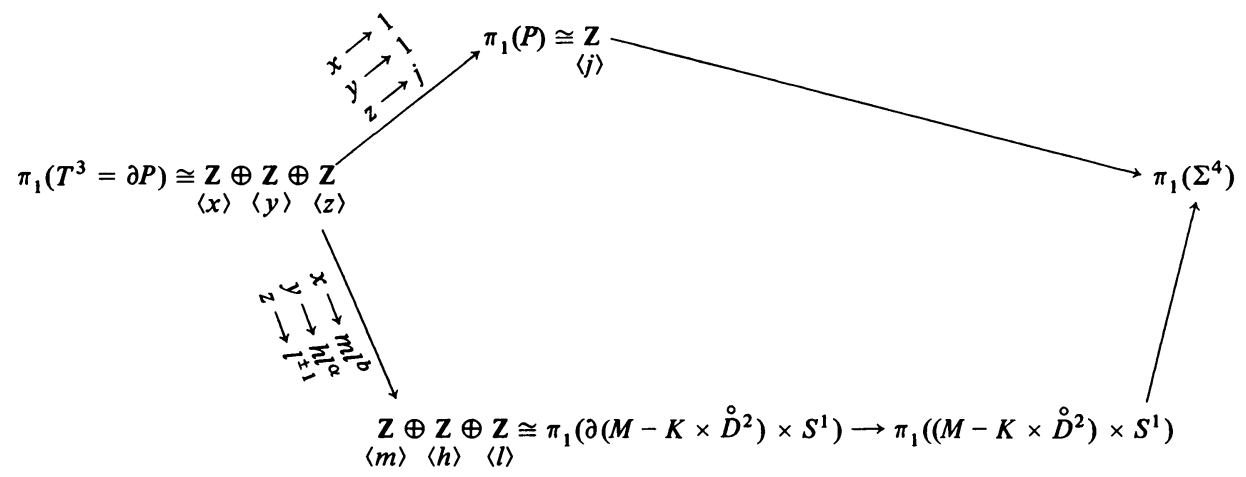

where $b$ and $\alpha$ are arbitrary integers.

By Van Kampen's theorem, we have

$$
\begin{aligned}
\pi_{1}\left(\Sigma^{4}\right) \cong\left\langle\pi_{1}\left(M^{3}-K \times \check{D}^{2}\right), h, j\right|[h, \sigma]=m l^{b}=h l^{\alpha}=j l^{ \pm 1}=1, \\
\left.\quad \forall \sigma \in \pi_{1}\left(M^{3}-K \times D^{2}\right)\right\rangle \\
\cong\left\langle\pi_{1}\left(M^{3}-K \times \check{D}^{2}\right), h \mid[h, \sigma]=m l^{b}=h l^{\alpha}=1, \forall \sigma\right\rangle \\
\cong\left\langle\pi_{1}\left(M^{3}-K \times \check{D}^{2}\right) \mid\left[l^{\alpha}, \sigma\right]=m l^{b}=1, \forall \sigma\right\rangle .
\end{aligned}
$$

The notation is a bit informal here. We suppress the generators and relations for the knot group. We shall often take the liberty of writing this as $\left\langle\pi_{1}\left(M-K \times \check{D}^{2}\right)\right| l^{\alpha}$ central, $\left.m l^{b}=1\right\rangle$. The notation " $l^{\alpha}$ central", of course, refers to the relations $\left[l^{\alpha}, \sigma\right]$, $\forall \sigma \in \pi_{1}\left(M-K \times D^{2}\right)$.

Now that we have arranged for $H_{1}(\Sigma)=0$ and computed $\pi_{1}(\Sigma)$, everything else goes nicely. By Poincaré duality and the Universal Coefficient Theorem [Sp] we have that $0=H_{1}(\Sigma) \cong H^{3}(\Sigma)$ and $H_{3}(\Sigma)=H^{1}(\Sigma)=0$. Therefore, $H_{2}(\Sigma)$ is free abelian, 
again by Universal Coefficients. We now have

$$
\begin{array}{ccc}
0 \rightarrow H_{2}\left(T^{3}\right) \stackrel{\phi}{\rightarrow} & H_{2}\left(\left(M-K \times \stackrel{\circ}{D}^{2}\right) \times S^{1}\right) & \oplus H_{2}(P) \rightarrow H_{2}(\Sigma) \rightarrow 0 \\
\| l & \| l & \| l \\
\mathbf{Z}^{3} & \mathbf{Z} & \mathbf{Z}^{2}
\end{array}
$$

Since $H_{2}(\Sigma)$ is free, $\phi$ must be an isomorphism, forcing $H_{2}(\Sigma)=0$, and $\Sigma$ is therefore a homology 4-sphere. We have proved

THEOREM 2.1. Given a knot $K$ in a homology 3-sphere $M^{3}$, and two integers $b, \alpha$, there exists a homology 4-sphere $\Sigma^{4}$ with

$$
\pi_{1}\left(\Sigma^{4}\right) \cong\left\langle\pi_{1}\left(M-K \times \stackrel{\circ}{2}^{2}\right) \mid 1=m l^{b}=\left[l^{\alpha}, \sigma\right], \forall \sigma \in \pi_{1}\left(M-K \times \stackrel{\circ}{ }^{2}\right)\right\rangle .
$$

REMARK. How close is this construction to Fintushel's? If we were trying to make the glueing equivariant, we would put an $S^{1}$ action on $P$, with quotient space a solid torus. Over a meridional disk will be a solid torus, corresponding to equator $\times D^{2}$ in one copy of $S^{2} \times D^{2}$. The fundamental group of the boundary of this solid torus is generated by $x$ and $y$ (recall $x$ and $y$ die in $\pi_{1}(P)$, while $z$ "goes around" $P$ ). We may put an $S^{1}$ action on $P$ so that $y$ is an orbit, $x$ is a section to the action over a meridian downstairs, and $z$ is a section over the longitude downstairs. The identification $x \mapsto m l^{b}$ is perfectly fine. Downstairs, we are glueing the solid torus into the knot complement, sending a meridian to a meridian plus $b$ longitudes, i.e., doing a Dehn surgery on $K$ with surgery coefficient $1 / b$ (see [R]). The identification $z \mapsto l^{ \pm 1}$ is also fine. Downstairs, up to orientation, we are matching longitudes. The identification $y \mapsto h l^{\alpha}$ is not fine. We are not matching orbits unless $\alpha=0$. The "nonequivariance" factor is given by $\alpha$.

If $\alpha=0$, we have glued equivariantly. There is an $S^{1}$ action on $\Sigma$ with quotient space equal to the homology sphere obtained from $M^{3}$ by $1 / b$ surgery on $K$. When the knot $K$ is clear from context, we will denote this space simply by $M_{b}$. The fundamental group of $M_{b}$ is given by $\pi_{1}\left(M_{b}\right) \cong\left\langle\pi_{1}\left(M-K \times D^{2}\right) \mid 1=m l^{b}\right\rangle$. Notice that this is exactly $\pi_{1}(\Sigma)$. This should not be surprising in view of the discussion in $\S 1$. Indeed, $\Sigma$ is just a spun $M_{b}$.

Suppose we form $M_{b} \times S^{1}$. Let $h=* \times S^{1}$, and surger the curve $h l^{\alpha}$. This produces a homology 4-sphere with the same fundamental group as in Theorem 2.1, and it is plausible that the two constructions are the same. This is almost true. Choosing explicit coordinates for $P$ gives a basis for $H_{1}\left(\partial P=T^{3}\right)$, and the above construction can be described by an element of GL(3,Z). Both $\left(M-K \times \check{D}^{2}\right) \times S^{1}$ and $P$ admit self-homeomorphisms which allow us to simplify this matrix to a particularly simple form, which yields the following description of these homology spheres:

THEOREM 2.2. The homology sphere of Theorem 2.1 is the result of surgery (with one of two possible framings) on the curve $h l^{\alpha}$ in $M_{b^{\prime}} \times S^{1}$, where $b^{\prime}=b(\bmod \alpha)$. 
The integer $b^{\prime}$ can be found in any given example if one is willing to go through the matrix simplifications alluded to above. It is not hard to see that the fundamental groups of these homology spheres depend only upon the equivalence class of $b$ $(\bmod \alpha)$, as Theorem 2.2 suggests. ${ }^{1}$

We do not need Theorem 2.2 for what follows, so we omit the proof. Notice that the two 2-sphere cores of $P$ determine knots in homology 4-spheres. It turns out that these include an interesting class of fibered knots in $S^{4}$ which generalize twist-spun knots. To describe them, we need the explicit matrix description and the proof of Theorem 2.2. This will appear in [P12].

3. The case of the missing torus knot. We now specialize to torus knots in $S^{3}$. Using the theory of Seifert manifolds (see [OR1] or [NR]), we shall be able to completely describe this situation. As usual, we try to glue $P$ to $\left(S^{3}\right.$-torus knot $\times$ $\left.D^{2}\right) \times S^{1}$ "nonequivariantly", but the presence of a circle action on the knot complement will make this impossible.

It will be convenient to use unnormalized Seifert invariants. For a review of this, see $[\mathbf{N R}]$.

The manifolds obtained by Dehn surgery on torus knots are completely classified (see [Mo]). We shall consider Dehn surgery of type $1 / b$ only. Let $K$ be a right-handed $(r, s)$-torus knot, where $r, s>0,(r, s)=1$. Writing $S^{3}$ as $\left\{\left(z_{1}, z_{2}\right)|| z_{i} \mid \leqslant 1, i=\right.$ $1,2\}$, give $S^{3}$ the $S^{1}$ action defined by $e^{i \theta} \cdot\left(z_{1}, z_{2}\right)=\left(e^{i r \theta} z_{1}, e^{i s \theta} z_{2}\right)$. Then $K$ is a principal orbit of this action, and $S^{3}$ has Seifert invariants $(0 ;(r, u),(s, v))$, where $r v+s u=1$. We need

LEMMA 3.1. Two principal fibers of the above $S^{1}$ action have linking number $=r s$.

Proof. Given two principal fibers, deform one to wrap $r$ times around the singular fiber of type $(r, u)$, and deform the other to wrap $s$ times around the singular fiber of type $(s, v)$. These two singular fibers form a trivial link in $S^{3}, \oslash$, and thus have linking number $=1$ (where we have picked orientation to assume +1$)$. Since linking is bilinear, this completes the proof.

It is standard to use the symbol $h$ for a principal orbit. Unfortunately, we have already used $h$, so let $i$ denote a principal orbit. Now do $1 / b, b \in \mathbf{Z}$, surgery on the torus knot $K$. We have the following, presumably well-known, lemma.

LEMMA 3.2. The result of $1 / b$ surgery on an $(r, s)$ torus knot is a Seifert homology 3-sphere, $\Sigma(r, s, \mid$ brs $-1 \mid)$, with three exceptional orbits of type $r, s$, and $\mid$ brs $-1 \mid$.

Proof. Since the torus knot is a principal orbit, a meridian $m$ of a tubular neighborhood will be a section to the action. We have shown that an orbit on the boundary of the tubular neighborhood has linking number $r s$ with the knot, so therefore a preferred longitude $l=i m^{-r s}$. Remove this tubular neighborhood and glue in a solid torus by sending a meridian to $m l^{b}=i^{b} m^{1-b r s}$. This is exactly how one creates an exceptional orbit of type $(|b r s-1|,-|b|)$. The result of the surgery will therefore have Seifert invariants $(0 ;(r, u),(s, v),(|b r s-1|,-|b|))$. The order of the first homology group is given by $u s|b r s-1|+v r|b r s-1|-|b| r s= \pm 1$,

\footnotetext{
'ADDED IN PROOF. In fact, the manifold itself only depends on $\alpha$ and the equivalence class of $b(\bmod \alpha)$. See [P12].
} 
giving a homology sphere as expected. As a final check, notice that +1 surgery on a right-handed trefoil knot $((r, s)=(2,3))$ yields $\Sigma(2,3,5)$, so our \pm signs are correct.

Now construct a homology 4 -sphere $\Sigma$ as described above. First we examine fundamental groups. We have

$$
\left.\pi_{1}(\Sigma) \cong\left\langle\pi_{1} \Sigma(r, s,|b r s-1|)\right| l^{\alpha} \text { central }\right\rangle .
$$

The fundamental group of $\Sigma(r, s,|b r s-1|)$ is well known [OR1] to be given by

$$
\left.\left\langle q_{1}, q_{2}, q_{3}, i\right| i \text { central, } 1=q_{1} q_{2} q_{3}=q_{1}^{r} i^{u}=q_{2}^{s} i^{v}=q_{3}^{b r s-1 \mid} i^{-|b|}\right\rangle,
$$

where the $q_{j}$ are sections to the action about exceptional orbits, and $i$ represents the class of a principal orbit. In the construction of $\Sigma(r, s,|b r s-1|)$, the longitude $l$ becomes the third exceptional orbit, denoted $t_{3}$. We have that $q_{3}=t_{3}^{|b|}$ and $i=t_{3}^{|b r s-1|}$ [OR1], so that $t_{3}=\left(i q_{3}^{-r s}\right)^{ \pm 1}$, depending on whether $b$ is negative or positive.

Thus, we see that $\pi_{1}\left(\Sigma^{4}\right) \cong\left\langle q_{1}, q_{2}, q_{3}, i\right| i$ central,

$$
\begin{array}{r}
\left.1=q_{1} q_{2} q_{3}=q_{i}^{r} i^{u}=q_{2}^{s} i^{v}=q_{3}^{|b r s-1|} i^{-|b|},\left(i q_{3}^{-r s}\right)^{ \pm \alpha} \text { central }\right\rangle \\
\cong\left\langle q_{1}, q_{2}, q_{3}, i\right| i \text { central, } q_{3}^{\alpha r s} \text { central, } \\
\left.1=q_{1} q_{2} q_{3}=q_{1}^{r} i^{u}=q_{2}^{s} i^{v}=q_{3}^{|b r s-1|} i^{-|b|}\right\rangle .
\end{array}
$$

This group may look new, but it is in fact a Seifert manifold group. To see this, divide by its center

$$
\begin{aligned}
\frac{\pi_{1}\left(\Sigma^{4}\right)}{\left\langle i, q_{3}^{\alpha r s}\right\rangle} & \cong\left\langle q_{1}, q_{2}, q_{3} \mid 1=q_{1} q_{2} q_{3}=q_{1}^{r}=q_{2}^{s}=q_{3}^{|b r s-1|}=q_{3}^{\alpha r s}\right\rangle \\
& \cong\left\langle q_{1}, q_{2}, q_{3} \mid 1=q_{1} q_{2} q_{3}=q_{1}^{r}=q_{2}^{s}=q_{3}^{\operatorname{gcd}(|b r s-1|, \alpha r s)}\right\rangle,
\end{aligned}
$$

which is a centerless Fuchsian group (see [Mag]). For the rest of this section, it will be convenient to define the following numbers.

$$
\begin{aligned}
\delta & =\operatorname{gcd}(|b r s-1|,|r s \alpha|)=\operatorname{gcd}(|b r s-1|,|\alpha|), \\
\delta x & =|b r s-1|, \quad \delta y=|\alpha|, \quad|b| m+n x=1 .
\end{aligned}
$$

The center of $\pi_{1}(\Sigma)$ is generated by $i$ and $q_{3}^{\delta}$. By using $i^{|b|}=q_{3}^{|b r s-1|}$, we see that

$$
\left(i^{n} q_{3}^{\delta m}\right)^{x}=i^{n x} q_{3}^{\delta m x}=i^{1-|b| m} q_{3}^{m \delta x}=i q_{3}^{-m|b r s-1|} q_{3}^{m|b r s-1|}=i
$$

and

$$
\left(i^{n} q_{3}^{\delta m}\right)^{|b|}=i^{n|b|} q_{3}^{m \delta|b|}=q_{3}^{n|b r s-1|+\delta m|b|}=q_{3}^{n \delta x+\delta m|b|}=q_{3}^{\delta} .
$$

So the center is actually cyclic, generated by $i^{n} q_{3}^{\delta m}$. It is now a simple matter to verify the following isomorphism:

$$
\begin{aligned}
& \left.\pi_{1}\left(\Sigma^{4}\right) \cong\left\langle q_{1}, q_{2}, q_{3}, i\right| 1=q_{1} q_{2} q_{3}=q_{1}^{r} i^{u}=q_{2}^{s} i^{v}=q_{3}^{|b r s-1|} i^{-|b|}, i^{n} q_{3}^{\delta m} \text { central }\right\rangle \\
& \begin{array}{ccc}
q_{i} & i \\
\downarrow & \downarrow \\
p_{i} & j^{x} & \cong \\
1 & \uparrow \\
p_{i} & j
\end{array} \\
& \left.\left\langle p_{1}, p_{2}, p_{3}, j\right| 1=p_{1} p_{2} p_{3}=p_{1}^{r} j^{u x}=p_{2}^{s} j^{v x}=p_{3}^{\delta} j^{-|b|}, j \text { central }\right\rangle .
\end{aligned}
$$


We have shown that $\Sigma^{4}$ has the same fundamental group as the Seifert homology sphere $\Sigma(r, s, \delta)$, which has Seifert invariants $(0 ;(r, u x),(s, v x),(\delta,-|b|))$. For future reference, notice what this above isomorphism does to $l^{\alpha}$ :

$$
\begin{aligned}
l^{\alpha} & =\left(i q_{3}^{-r s}\right)^{ \pm \alpha} \mapsto\left(j^{x|\alpha|} p_{3}^{-r s|\alpha|}\right)^{ \pm 1}=\left(j^{x|\alpha|} p_{3}^{-r s \delta y}\right)^{ \pm 1} \\
& =\left(j^{x|\alpha|-r s y|b|}\right)^{ \pm 1}=\left(j^{x \delta y-r s y|b|}\right)^{ \pm 1}=j^{ \pm y(|b r s-1|-|b| r s)}=j^{ \pm y}
\end{aligned}
$$

The reason we are not getting a new group is because we are not getting a new manifold. Since $S^{3}-K \times \dot{D}^{2}$ has an $S^{1}$ action, there are many $S^{1}$ actions on $\left(S^{3}-K \times \check{D}^{2}\right) \times S^{1}$, and it is actually impossible to glue $P$ to $\left(S^{3}-K \times \check{D}^{2}\right) \times S^{1}$ "nonequivariantly". We omit these calculations (see [PI1]), since we do not need this information for what follows, and summarize the state of our knowledge:

THEOREM 3.3. Let $K$ be the $(r, s)$ torus knot in $S^{3}$. Attach $P$ to $\left(S^{3}-K \times D^{2}\right) \times S^{1}$ to construct a homology 4-sphere $\Sigma$ with

$$
\left.\pi_{1} \Sigma \cong\left\langle\pi_{1}\left(S^{3}-K \times D^{2}\right)\right| 1=m l^{b}, l^{\alpha} \text { central }\right\rangle \cong \pi_{1} \Sigma(r, s, \delta) .
$$

Then there is an $S^{1}$ action on $\Sigma$, and $\Sigma$ is a spun $\Sigma(r, s, \delta)$.

4. Homology spheres without $S^{1}$ actions. In this section we show that we can get new fundamental groups and manifolds with our construction. We shall construct homology 4-spheres $\Sigma$ (more generally, $\Sigma$ satisfying $H_{1}(\Sigma ; \mathbf{Z})=0$ ) that do not support $S^{1}$ actions, and whose fundamental groups cannot be those of 3-manifolds.

Recall from $\S 1$ that if $S^{1}$ acts effectively on $\Sigma$, and $H_{1}(\Sigma ; Z)=0$, then $\Sigma$ is a connected sum of copies of $S^{2} \times S^{2}$ and $\pm \mathrm{CP}(2)$ with a spun homology sphere $N^{3}$. In particular, $\pi_{1}\left(\Sigma^{4}\right) \cong \pi_{1}\left(N^{3}\right)$. All we have to do is produce $\Sigma^{4}$ with fundamental groups that could not be 3-manifold groups.

To do this, we invoke some powerful theorems of 3-manifold theory. (See [H] for definitions and proofs.) Suppose a group $\pi$ has nontrivial center and is the group of a homology 3-sphere $N^{3}$. Write $N$ uniquely as a connected sum of irreducible manifolds $N_{1} \# \cdots \# N_{n}$. Since $\pi_{1} N$ has center, all but one of the $N_{i}$ must be homotopy spheres, and the $N_{i}$ with nontrivial $\pi_{1}$ must have $\pi_{1} N_{i}=\pi_{1} N$. Therefore, $\pi$ is the group of an irreducible homology 3-sphere $N_{i}$. If $\pi$ is infinite, a standard 3-manifold argument shows that $N_{i}$ is aspherical, and therefore we have

$$
H_{*}(\pi ; \mathbf{Z}) \cong H_{*}(K(\pi, 1) ; \mathbf{Z}) \cong H_{*}\left(N_{i} ; \mathbf{Z}\right) \cong H_{*}\left(S^{3} ; \mathbf{Z}\right) .
$$

If we produce groups $\pi$ with homology in dimension 4 , we are done.

The construction of $\Sigma^{4}$ produces center in $\pi_{1}$. The problem is that for a knot picked at random, the group $\left\langle\pi_{1}\left(M-K \times \check{D}^{2}\right)\right| l^{\alpha}$ central, $\left.1=m l^{b}\right\rangle$ is not readily understandable. Conceivably, the relations $\left[l^{\alpha}, x\right]=1$ might trivialize the group (e.g., if $b \equiv 0(\alpha)$ ). The torus knot situation was eminently computable, but did not produce new groups. So the idea is simply to embed the torus knots in homology spheres in a trivial fashion, and deal with them not where they normally live, namely $S^{3}$, but in $S^{3} \#$ homology sphere, thereby destroying the $S^{1}$ action on the knot complement. 
With this in mind, let $M$ be any homology 3-sphere with $\pi_{1} M \neq 1$. In a small ball $B^{3}$ in $M$, draw an $(r, s)$ torus knot $K$ and construct $\Sigma^{4}$ as usual.

$$
\Sigma^{4}=P \underset{T^{3}}{\cup}\left(M-K \times D^{2}\right) \times S^{1},
$$

and

$$
\left.\pi_{1}(\Sigma) \cong\left\langle\pi_{1}\left(M-K \times D^{2}\right)\right| 1=m l^{b}, l^{\alpha} \text { central }\right\rangle .
$$

The preferred longitude $l$ is unchanged by embedding $K$ into $M$. Since $1 / b$ surgery on $K$ will obviously produce $\Sigma(r, s,|b r s-1|) \# M$, we can write $\pi_{1}(\Sigma) \cong$ $\left\langle\pi_{1} \Sigma(r, s,|b r s-1|) * \pi_{1} M\right| l^{\alpha}$ central $\rangle$. Note that $l^{\alpha}$ commutes with $\pi_{1} M$ as well as with $\pi_{1} \Sigma(r, s,|b r s-1|)$.

REMARK. Before analyzing $\pi_{1}(\Sigma)$, note that we can construct the same group another way. The plumbing is attached to $\partial\left(K \times D^{2}\right) \times S^{1}$, and basically ignores $M$. So first think of this construction as going on inside $S^{3}$. From Theorem 3.3, we see that this creates a spun $\Sigma(r, s, \delta)$. To embed this in $M$, we must remove $B^{3} \times S^{1}$ from the spun $\Sigma(r, s, \delta)$, where the $S^{1}$ corresponds to $h \simeq l^{-\alpha}$. Now add in $\left(M-B^{3}\right) \times S^{1}$ to create $\Sigma$. $\left(M-B^{3}\right) \times S^{1}$ is the complement of a knot in a spun $M$, hence a homology circle. What we have just done is

$$
\Sigma^{4}=\left(\operatorname{spun} \Sigma(r, s, \delta)-S^{1} \times B^{3}\right) \underset{S^{1} \times S^{2}}{\cup}\left(M^{3}-B^{3}\right) \times S^{1},
$$

i.e., remove a circle from a homology sphere and replace by a homology circle. By Van Kampen's theorem,

$$
\begin{aligned}
\pi_{1}\left(\Sigma^{4}\right) & \cong\left\langle\pi_{1} \Sigma(r, s, \delta) *\left(\pi_{1} M \times \mathbf{Z}(\bar{h})\right) \mid \bar{h}=l^{\alpha}\right\rangle \\
& \left.\cong\left\langle\pi_{1} \Sigma(r, s, \delta) * \pi_{1} M\right| l^{\alpha} \text { central }\right\rangle .
\end{aligned}
$$

In any case, we have constructed $\Sigma^{4}$. Recall that the isomorphism

$$
\left.\left\langle\pi_{1} \Sigma(r, s,|b r s-1|)\right| l^{\alpha} \text { central }\right\rangle \stackrel{\cong}{\rightarrow} \pi_{1} \Sigma(r, s, \delta)
$$

takes the element $l^{\alpha}$ to $i^{ \pm y}$, where $i$ generates the center of $\pi_{1} \Sigma(r, s, \delta)$, and $\delta y=|\alpha|, \delta=\operatorname{gcd}(|b r s-1|,|\alpha|)$. Therefore, we can write $\pi_{1}\left(\Sigma^{4}\right) \cong$ $\left\langle\pi_{1} \Sigma(r, s, \delta) * \pi_{1} M\right| i^{ \pm y}$ central $\rangle$. Assume $\delta \neq 1$.

We now determine some of the structure of $\pi_{1}(\Sigma)$. Dividing by the normal closure of $\pi_{1} M$, we get

$$
\begin{aligned}
\pi_{1}(\Sigma) & \left.\rightarrow\left\langle\pi_{1} \Sigma(r, s, \delta) * \pi_{1} M\right| i^{ \pm y} \text { central, } x=1, \forall x \in \pi_{1} M\right\rangle \\
& \left.\cong\left\langle\pi_{1} \Sigma(r, s, \delta)\right| i^{ \pm y} \text { central }\right\rangle \cong \pi_{1} \Sigma(r, s, \delta) .
\end{aligned}
$$

For the moment, exclude $\{r, s, \delta\}=\{2,3,5\}$. Then it is well known [O] that the center of $\pi_{1} \Sigma(r, s, \delta)$ is infinite cyclic, generated by $i$. Since $i^{ \pm y}$ generates a copy of $\mathbf{Z}$ in a quotient of $\pi_{1} \Sigma$, it generates a copy of $\mathbf{Z}$ in $\pi_{1} \Sigma$. Dividing by this normal subgroup, we have

$$
1 \rightarrow \mathbf{Z} \rightarrow \pi_{1}(\Sigma) \rightarrow\left\langle\pi_{1} \Sigma(r, s, \delta) * \pi_{1} M \mid i^{y}=1\right\rangle \rightarrow 1
$$

or

$$
1 \rightarrow \mathbf{Z} \rightarrow \pi_{1}(\Sigma) \rightarrow \pi_{1} \Sigma(r, s, \delta) /\left\langle i^{y}\right\rangle * \pi_{1} M \rightarrow 1,
$$

a central extension. 
As we have pointed out, since $\pi_{1} \Sigma$ has center and is infinite, the presence of an $S^{1}$ action on $\Sigma$ would force $\pi_{1} \Sigma$ to be the fundamental group of an aspherical homology 3-sphere, say $N^{3}$. Writing $M=M_{1} \# \cdots \# M_{m}$ as a sum of prime homology spheres, suppose that some $M_{i}$ is aspherical. Pull back the above extension via the natural inclusion $\pi_{1} M_{i} \hookrightarrow \pi_{1} \Sigma(r, s, \delta) /\left\langle i^{y}\right\rangle * \pi_{1} M$ to get $\mathbf{Z} \times \pi_{1} M_{i}$ as a subgroup of $\pi_{1} \Sigma$ (since $H^{2}\left(M_{i}\right)=0$, there are no nontrivial central extensions). The homology of $\mathbf{Z} \times \pi_{1} M_{i}$ can be computed from a covering space of $N^{3}$ and is therefore trivial above dimension 3. But this is absurd, since $H_{4}\left(\mathrm{Z} \times \pi_{1} M_{i}\right) \cong \mathbf{Z}$. If any $\pi_{1} M_{i}$ is finite, we have an immediate contradiction, since $\pi_{1} \Sigma$ will contain torsion.

Finally, the problem with $\{r, s, \delta\}=\{2,3,5\}$ is that the center of $\pi_{1} \Sigma(2,3,5)$ is just $\mathbf{Z}_{2}$. If we accidentally pick $y$ even, then $i^{y}=1$ and we get $\pi_{1} \Sigma \cong$ $\pi_{1} \Sigma(2,3,5) * \pi_{1} M$, which is certainly a 3 -manifold group. If $y$ is odd, however, we get a $\mathbf{Z}_{2}$ center, yielding $\pi_{1} \Sigma$ as an infinite group with center and with torsion, which we have already pointed out cannot be the group of a 3-manifold.

We have proved

THEOREM 4.1. Let $M^{3}$ be any homology 3-sphere with $\pi_{1} M \neq 1$. Pick an $(r, s)$ torus knot, pick $b$ and $\alpha$ so that $\operatorname{gcd}(|b r s-1|, \alpha)=\delta \neq 1$, and let $y \delta=|\alpha|$. If $\{r, s, \delta\}=$ $\{2,3,5\}$, we demand that $y$ is odd. Then the plumbing construction will produce $a$ homology 4-sphere $\Sigma$ with $\pi_{1}(\Sigma) \cong\left\langle\pi_{1} \Sigma(r, s, \delta) * \pi_{1} M\right| i^{y}$ central $\rangle$. $\Sigma$ does not admit an $S^{1}$ action, and $\pi_{1} \Sigma$ cannot be a 3-manifold group.

Generalizations. (1) If we take a connected sum of one of the above $\Sigma$ with any homology 4-sphere, the same result holds, since Kneser's conjecture [H] says that any splitting of a 3-manifold group as a free product must reflect a splitting of the manifold as a connected sum.

(2) Since the same criterion on $\pi_{1}$ holds for $\Sigma^{4}$ satisfying $H_{1}(\Sigma)=0$ by Theorem 1.2 , connected sums of manifolds in the above theorem with standard simply connected 4-manifolds such as $S^{2} \times S^{2}$ or $\pm \mathrm{CP}(2)$ will also yield 4-manifolds without $S^{1}$ actions.

Question. As remarked earlier, Kervaire showed that $H_{1}(G)=H_{2}(G)=0$ are necessary and sufficient conditions for a finitely presented group $G$ to be $\pi_{1}\left(\Sigma^{n}\right)$, $n \geqslant 5$. If $n=4$, and we make the stronger assumption that $H_{1}(G)=0$ and $G$ has a presentation with an equal number of generators and relations, Kervaire constructs $\Sigma^{4}$. Also, fundamental groups of homology 3-spheres have such presentations. It seems reasonable to conjecture that the groups in the above theorem do not have such presentations. Can this be proved?

\section{REFERENCES}

[F1] R. Fintushel, Locally smooth circle actions on homotopy 4-spheres, Duke Math. J. 43 (1976), 63-70. , Circle actions on simply connected 4-manifolds, Trans. Amer. Math. Soc. 230 (1977), $147-171$.

[F3] $377-390$.

[H] J. Hempel, 3-manifolds, Princeton Univ. Press, Princeton, N. J., 1976.

[K] M. Kervaire, Smooth homology spheres and their fundamental groups, Trans. Amer. Math. Soc. 144 (1969), 67-72.

[Mag] W. Magnus, Noneuclidean tesselations and their groups, Academic Press, New York, 1974. 
[Mo] L. Moser, Elementary surgery along a torus knot, Pacific J. Math. 38 (1971), 737-745.

[NR] W. D. Neumann and F. Raymond, Seifert manifolds, plumbing, $\mu$-invariant, and orientation reversing maps, Conf. on Algebraic and Geometric Topology (Santa Barbara, 1978), Lecture Notes in Math., vol. 664, Springer-Verlag, Berlin and New York, 1979, pp. 162-196.

[O] P. Orlik, Seifert manifolds, Lecture Notes in Math., vol. 291, Springer-Verlag, Berlin and New York, 1972.

[OR1] P. Orlik and F. Raymond, Actions of SO(2) on 3-manifolds, Proc. Conf. Transformation Groups (New Orleans, 1967), (Paul Mostert, editor), Springer-Verlag, Berlin and New York, 1968, pp. 297-318.

[P] P. S. Pao, Non-linear circle actions on the 4-sphere and twisting spun knots, Topology 17 (1978), 291-296.

[PI1] S. Plotnick, Knots, automorphisms, and homology 4-spheres, Thesis, Univ. of Michigan, 1979.

[PI2] , Fibered knots in $S^{4}$-twist spinning, rolling, and other operations (in preparation).

[R] D. Rolfson, Knots and links, Publish or Perish, Cambridge, Mass., 1976.

[Sp] E. H. Spanier, Algebraic topology, McGraw-Hill, New York, 1966.

Department of Mathematics, University of Chicago, Chicago, Illinois 60637

Current address: Department of Mathematics, Columbia University, New York, New York 10027 